\title{
The Intergenerational Transmission of Values in National and Immigrant Families: The Role of Zeitgeist
}

\author{
Paul Vedder · John Berry $\cdot$ Colette Sabatier · \\ David Sam
}

Received: 21 July 2008/Accepted: 19 November 2008/Published online: 9 December 2008

(C) The Author(s) 2008. This article is published with open access at Springerlink.com

\begin{abstract}
Correspondence in value orientation between parents and their offspring may be due to actual transmission processes between generations, but it may also be due to influences from the general value context in society that are common to parents and their offspring. This common value context is referred to as Zeitgeist. The present study deals with one family relationship value (i.e., parents' and adolescents' obligations toward the family). Participants were 1,252 immigrant and 726 national adolescent-parent dyads from 10 Western countries. There were significant relationships between the value placed on family obligations among parents and offspring, and these were independent of gender. Zeitgeist effects, both intergenerational and intragenerational, were found. The strength of these Zeitgeist effects depended on the basis for defining Zeitgeist, either a person's own ethnic group or the wider community including both nationals and immigrants. For explaining national adolescents' acceptance of their family obligations, both the ethnic and the national Zeitgeist played a role, whereas in the immigrant groups only the ethnic Zeitgeist played a significant role. In short, in an immigration context it makes sense to distinguish the
\end{abstract}

P. Vedder $(\bowtie)$

Department of Education and Child Studies, Leiden University,

P.O. Box 9555, 2300 RB Leiden, The Netherlands

e-mail: Vedder@fsw.leidenuniv.nl

J. Berry

Queens University, Kingston, Canada

C. Sabatier

Université Victor Segalen, Bordeaux, France

D. Sam

Bergen University, Bergen, Norway influence of a person's own ethnic group from the influence of the wider community, including other ethnic groups. Explanations are suggested and implications are discussed.

Keywords Values $\cdot$ Immigrant youth $\cdot$ Zeitgeist

In this article, we examine the process and outcomes of the transmission of values to adolescents. The continuity of values between generations is an important goal of socialization and is crucial for the functioning of society (Fuligni and Zhang 2004; Schönpflug 2001). This continuity depends on the effectiveness of transmission processes. Not all values are transmitted to the same degree, nor do all values have comparable transmission efforts. There are variations across types of values: collectivist values appear to be more often transmitted than individualist ones (Knafo and Schwartz 2001). Earlier, Schwartz (1992) clarified that values that are commonly shared by members of a society lead to little explicit effort by parents to reach value correspondence between them and their offspring, whereas values less endorsed in the community lead to more efforts by parents to reach correspondence.

This article tests a model proposed by Boehnke (2001). $\mathrm{He}$ proposed that the correspondence in values between generations within families in a society are due to a variety of influences amongst which are at least two contextual influences: parental influence on their offspring and the influence exerted by the sharing of the values and ideas prevailing in the social environment of each generation. $\mathrm{He}$ called this latter common value environment the "Zeitgeist", and used it to show that co-variation in scores for particular children and their parents can in part be 
explained by a general or modal social value climate that leads to a correlation between generations, or between persons within generations, even when the persons are nonkin. Using another methodology Boehnke et al. (2007) reported a similar finding.

The study of value transmission processes is important for understanding child development and it clarifies that the parent-child relationship is not the only important relationship for this development. The child's development is affected by other influences (e.g., the Zeitgeist) that may impact both parents and children (or just the children) in ways that are beyond parental control. From a developmental perspective a relevant question is who represents the Zeitgeist. Boehnke et al. (2007) refer to a common Zeitgeist which they consider to be the same for all members of a particular group in a particular time frame. Perhaps such an omnipresent Zeitgeist may exist as an abstract entity that can be operationalized as an average score on a value preference list in a particular group or representative sample. However, value transmission is based on interactions that take place between persons. In the exploration of the transmission of values to youth the question is: who are these persons? in this paper we explore first whether adolescents' and parents' value for family obligations are correlated and second, whether, beyond this correlation, the value placed on obligations by adolescents and their parents can be linked to the same value among either non-kin adolescents (henceforth mock peers) or nonkin adults (henceforth mock parents), or both (i.e., the Zeitgeist).

Harris $(1995,1998)$ argues in her controversial group socialization theory that children's experiences with groups of peers determine their socialization into the larger culture, and that, beyond infancy, parents make very little contribution to the socialization of their children. Dyadic relations with parents, siblings, and teachers according to the theory have minimum effects on psychological characteristics in adulthood (Vandell 2000). If indeed we would find that the Zeitgeist represented by non-kin peers does contribute more to the explanation of variance in adolescents' value preferences than non-kin adults do, this could be interpreted as support for Harris' argument.

\section{Value Transmission in an Immigration Context}

In an immigration context, parents and adolescents are involved in an acculturation process. During this process, they usually have different experiences of the two cultures, and have different future expectations (Berry 2006; Bornstein and Cote 2006; Kwak and Berry 2001; Szapocznik and Kurtines 1993). Following immigration, there are clear messages promoting the values of both the heritage culture and those of the society of settlement. The maintenance of traditional cultural values and customs often have a strong emotional value for individuals: their continuity may be promoted by involvement in the same-ethnic social network made up of parents, other same-ethnic adults, ethnocultural institutions, and peers. The experiences with the society of settlement are especially salient for adolescents through their school and peer group relationships within the larger society (Birman et al. 2002). Although families belonging to the societies of settlement do not have the same experience, they are usually affected by contacts with members of immigrant groups. As a consequence they may become more aware of the need to transmit particular values to their children, such as values related to the national culture or to cultural diversity (cf. Aboud and Doyle 1996; Carlson and Iovini 1985).

Boehnke et al. tested their model of cultural transmission with a sample of national residents only. In this article, we present analyses using data from both immigrant and national samples selected from a large scale study on the acculturation and adaptation of immigrant and national and youth and their parents in ten western countries (Berry et al. 2006). An important question is: which social value climate is most influential as the Zeitgeist? Boehnke et al. (2007) suggest that it is the broad societal, multiethnic modal value climate. However, it may also be the modal value climate within one's own ethnic group. The present study explores the role of both the broad societal Zeitgeist and the Zeitgeist based on the particular ethnic group, either national or immigrant. This question can also be phrased whether or not immigrants are influenced by the value preferences of nationals, and whether nationals are influenced by the value preferences of the immigrants. If adolescents' and parents' value preferences can be explained by Zeitgeist influences from their own groups, but not by the value orientation of the wider community, this may indicate a lack of mutual understanding, respect, and social cohesion both between and within groups.

\section{Family Relationship Values}

In all cultures, parents socialize their children into the values and practices of their society. Immigrant parents bring with them the values of their culture of origin and strive to preserve them in their new environment and teach them to their children. In many cases the values they hold differ from those of the society of settlement. There is evidence that members of non-European cultures hold stronger values related to family interdependence and respect toward parents than do people of north and west European origin (Fuligni et al. 2002; Georgas et al. 2006). Immigrants from such backgrounds continue to emphasize 
these values (Phalet and Schönpflug 2001; Phinney et al. 2005). Adolescents from cultures that emphasize family interdependence consider their household chores to be communal activities. These adolescents spent more time with adults on activities supervised by adults than adolescents from Western societies (Fuligni et al. 2002). In contrast, Western adolescents spend more time with peers on activities of their own choice and unsupervised by adults. Household chores or other communal activities that require time and effort and are non-preferred activities, are seen as obligations that have to compete for time and attention with activities of adolescents' own choice and liking. Parents' wish for their children to comply with such obligations is confronted with adolescents' wish to be materially compensated (cf. Bowes et al. 2001; Kagitcibasi 2007). Hence, communal obligations may change into individual claims to material rewards during the process of acculturation.

In this study we focus on parents' and adolescents' acceptance or appreciation of obligations of adolescent children toward their family and parents. Transmission of family obligations can be seen both as part of civic socialization and as a way to secure family solidarity (Fuligni et al. 2002). As a result of differences between the cultures of immigrants and the cultures of the society of settlement, immigrant parents face the task of teaching their children to accept family related obligations (that represent a notion of Zeitgeist typical of their ethnic group) while living in a new society that often holds different values (characterized by another Zeitgeist). Their children are exposed to the values of their parents but also to the values of the larger society in which they live. As a result, the children may adhere less strongly to their parents' values and feel more attracted to the Zeitgeist typical of the society of settlement. Although much of the preceding text suggests that we focus on value transmission from parents to their children, we actually do not study the direction of influences, but just correspondence. This means that we are interested in explaining adolescents' preference for family obligations as much as in explaining parents' preference. With respect to both adolescents and parents we explore the role of Zeitgeist (both ethnic and national) in explaining preferences for family obligations.

\section{Aim and Hypotheses}

Our aim in this article is to use a sample of immigrant and national parent-child dyads to examine the relationship between adolescents' and parents' acceptance of family obligations. Moreover, we explore the contribution of nonkin peers and non-kin adults to explaining variance in adolescents' and parents' family obligation preferences, distinguishing between an ethnic-specific Zeitgeist and a national Zeitgeist to the explanation of differences in preferences for family obligations.

In addition to examining these links between the appreciation or acceptance of obligations, we take an ecological approach (Berry and Georgas 2008). This ecological approach goes beyond examining the role of the value Zeitgeist. This approach addresses the role of social features and conditions of the lives of parents and children, such as parents' level of education, gender roles, and the proportion of life that immigrant adolescents have spent in their country of residence.

We test the following three hypotheses: (1) There is a significant relationship between the acceptance of the value of obligations toward the family held by adolescents and their parents; (2) beyond this relationship, there is a correspondence between the obligation scores of adolescents or parents and the Zeitgeist, represented by persons who are non-kin; and (3) the strength of this correspondence depends on the definition of Zeitgeist (that is, whether it refers to one's own ethnic group or to the broader society); however, we do not have any basis to suggest which Zeitgeist will make the greater contribution.

\section{Method}

\section{Sample}

The international study of immigrant youth sampled immigrant youth from 26 different cultural backgrounds who had settled in 13 countries (Berry et al. 2006). In each country, we sampled both national and immigrant youth between 13 and 18 years of age. For this study, we included only adolescents whose parents also participated in the study. The resulting sample included 1,252 immigrant adolescent-parent dyads and 726 national adolescent-parent dyads from 10 countries (Australia, Canada, Finland, France, Germany, Norway, Sweden, The Netherlands, UK, and the US) and 18 different ethnocultural groups (Table 1). In the immigrant group, $15 \%$ of all dyads were father-son dyads, $29 \%$ were mother-son dyads, $17 \%$ were father-daughter dyads and 39\% were mother-daughter dyads. A largely comparable distribution was found in the national sample: $12 \%$ father-son, $32 \%$ mother-son, $12 \%$ father-daughter, and $44 \%$ mother-daughter dyads. Hence more daughters than sons and more mothers than fathers participated. About $76 \%$ of the immigrant adolescents were either born in the country of settlement or born elsewhere, but arrived before the age of 7 (defined as 2nd generation immigrants). The other immigrant adolescents were first generation immigrants who were born in their country of origin and who came to their present country after the age 
Table 1 The countries and Ethnic groups that participated in the study, their size and other sample characteristics

\begin{tabular}{|c|c|c|c|c|c|c|c|c|c|c|}
\hline \multirow[t]{2}{*}{ Country } & \multirow[t]{2}{*}{ Group } & \multicolumn{6}{|c|}{ Adolescents } & \multicolumn{3}{|l|}{ Parents } \\
\hline & & $N$ & $\begin{array}{l}\text { Girls } \\
(\%)\end{array}$ & Age & $\begin{array}{l}\text { 2nd } \\
\text { genera } \\
(\%)\end{array}$ & $\begin{array}{l}\text { Life in } \\
\text { country } \\
(\%)\end{array}$ & Obligations & $\begin{array}{l}\text { Mothers } \\
(\%)\end{array}$ & SES* & Obligations \\
\hline \multirow[t]{4}{*}{ Australia } & Nationals & 98 & 58 & 15.2 & & 100 & 3.0 & 66 & 6.5 & 3.4 \\
\hline & Vietnamese & 48 & 75 & 16.0 & 74 & 73 & 3.6 & 63 & 4.9 & 4.2 \\
\hline & Chinese & 44 & 39 & 15.3 & 57 & 73 & 3.5 & 73 & 6.5 & 3.9 \\
\hline & Filipinos & 52 & 60 & 15.2 & 73 & 81 & 3.8 & 64 & 7.1 & 4.4 \\
\hline \multirow[t]{4}{*}{ Canada } & Nationals & 99 & 54 & 15.7 & & 100 & 3.2 & 56 & 5.7 & 3.4 \\
\hline & Vietnamese & 24 & 42 & 16.2 & 27 & 77 & 3.7 & 63 & 5.2 & 4.2 \\
\hline & Koreans & 30 & 27 & 15.9 & 41 & 61 & 3.7 & 47 & 6.7 & 3.9 \\
\hline & Indians & 27 & 37 & 15.5 & 89 & 91 & 3.7 & 33 & 7.4 & 4.0 \\
\hline \multirow[t]{3}{*}{ Finland } & Nationals & 39 & 39 & 15.6 & & 100 & 2.8 & 85 & 4.7 & 3.0 \\
\hline & Vietnamese & 63 & 61 & 15.3 & 29 & 51 & 3.8 & 51 & 4.0 & 4.0 \\
\hline & Turks & 22 & 73 & 16.4 & 86 & 91 & 2.9 & 91 & 5.5 & 3.2 \\
\hline \multirow[t]{6}{*}{ France } & Nationals & 24 & 63 & 16.2 & & 100 & 3.1 & 83 & 6.4 & 3.3 \\
\hline & Vietnamese & 21 & 71 & 16.4 & 86 & 91 & 3.4 & 67 & 7.1 & 3.8 \\
\hline & Turks & 24 & 50 & 15.8 & 100 & 100 & 3.7 & 71 & 3.9 & 4.2 \\
\hline & Algerians & 18 & 67 & 16.0 & 72 & 82 & 3.8 & 72 & 5.6 & 4.0 \\
\hline & Moroccans & 12 & 50 & 15.4 & 92 & 96 & 3.5 & 42 & 3.8 & 4.1 \\
\hline & Portuguese & 47 & 45 & 16.0 & 96 & 97 & 3.4 & 75 & 4.1 & 3.8 \\
\hline \multirow[t]{3}{*}{ Germany } & Nationals & 78 & 62 & 16.8 & & 100 & 2.9 & 65 & 6.0 & 3.0 \\
\hline & Turks & 8 & 13 & 16.1 & na & 97 & 2.9 & 38 & 3.8 & 3.9 \\
\hline & Portuguese & 11 & 73 & 17.1 & na & 86 & 3.0 & 64 & 4.9 & 3.5 \\
\hline \multirow[t]{5}{*}{ Norway } & Nationals & 89 & 48 & 15.0 & 100 & 100 & 2.8 & 90 & 6.3 & 3.1 \\
\hline & Vietnamese & 10 & 90 & 15.5 & 60 & 74 & 3.4 & 80 & 4.4 & 4.2 \\
\hline & Pakistanis & 8 & 63 & 15.3 & 88 & 95 & 4.0 & 25 & 5.1 & 4.2 \\
\hline & Turks & 8 & 63 & 15.0 & 50 & 66 & 3.8 & 13 & 3 & 4.0 \\
\hline & Chileans & 11 & 45 & 14.9 & 46 & 72 & 3.4 & 91 & 5.5 & 4.0 \\
\hline \multirow[t]{6}{*}{ Sweden } & Nationals & 64 & 59 & 16.0 & & 100 & 3.0 & 86 & 5.2 & 2.9 \\
\hline & Vietnamese & 27 & 56 & 15.8 & 70 & 80 & 3.6 & 93 & 3.1 & 3.7 \\
\hline & Turks & 51 & 61 & 15.0 & 88 & 93 & 4.0 & 75 & 3.8 & 4.1 \\
\hline & Kurds & 5 & 80 & 14.6 & 60 & 73 & 3.9 & 60 & 4.2 & 3.3 \\
\hline & $\begin{array}{l}\text { Latin } \\
\quad \text { Americans }\end{array}$ & 34 & 65 & 15.1 & 74 & 86 & 3.5 & 97 & 5.8 & 3.6 \\
\hline & Finns & 57 & 54 & 14.9 & 98 & 100 & 3.1 & 95 & 4.1 & 3.2 \\
\hline \multirow[t]{2}{*}{ UK } & Nationals & 43 & 49 & 15.5 & & 100 & 2.9 & 77 & 4.5 & 3.4 \\
\hline & Indians & 48 & 42 & 15.3 & 96 & 96 & 3.7 & 42 & 5.1 & 4.2 \\
\hline \multirow[t]{4}{*}{ USA } & Nationals & 108 & 62 & 14.6 & & 100 & 3.3 & 80 & 6.9 & 3.6 \\
\hline & Vietnamese & 64 & 64 & 15.1 & 77 & 86 & 3.7 & 61 & 4.7 & 4.3 \\
\hline & Armenians & 104 & 58 & 14.5 & 51 & 71 & 4.1 & 76 & 6.5 & 4.3 \\
\hline & Mexicans & 109 & 61 & 14.6 & 84 & 91 & 3.9 & 75 & 4.4 & 4.1 \\
\hline \multirow[t]{4}{*}{ Netherlands } & Nationals & 84 & 58 & 15.0 & & 100 & 2.6 & 82 & 6.2 & 2.8 \\
\hline & Turks & 116 & 53 & 14.7 & 98 & 99 & 3.8 & 49 & 4.0 & 4.0 \\
\hline & Surinamese & 75 & 51 & 15.1 & 97 & 99 & 3.5 & 75 & 4.8 & 3.9 \\
\hline & Antilleans & 74 & 61 & 15.0 & 62 & 76 & 3.5 & 88 & 4.5 & 3.8 \\
\hline \multirow[t]{2}{*}{ Total } & Nationals & 1,252 & 56 & 15.4 & 100 & & 3.0 & 75 & 6.0 & 3.2 \\
\hline & Immigrants & 726 & 56 & 15.2 & 85 & 76 & 3.7 & 68 & 5.0 & 4.0 \\
\hline
\end{tabular}

* SES was based on highest level of education of either parent (an eight point rating scale running from no primary school to [post] graduate degree) 
of 6 . The mean age of the national adolescents was 15.43 $(\mathrm{SD}=1.60)$ and of the immigrant adolescents 15.21 $(\mathrm{SD}=1.58)$. For each immigrant adolescent we calculated the proportion of life spent in the country of residence. For some ethnic groups, like the Turks in France and the Finns in Sweden, the average proportion was $100 \%$, indicating that all the participating Turkish and Finnish adolescents in these respective countries are second generation. Table 1 also presents information about the gender of the participating parents. In general, mothers were more frequently the participating parent. The highest rates were found in the national groups in Finland, Norway, and Sweden, but mothers were also overrepresented in some immigrant groups, such as the Vietnamese in Norway and Sweden, the Turks in Finland, and the Finns in Sweden. Furthermore, Table 1 presents mean obligation scores for adolescents and parents. The highest scores were reported by adolescents in the Turkish group in Sweden and in the Armenian group in the USA. The lowest scores were found in the national groups in the European countries. The latter was also the case for parental obligation scores, but the highest parental obligation scores were found amongst the Vietnamese in the USA, Australia, Canada, and Norway, Armenians in the USA, and Filipinos in Australia.

Parents also reported their highest achieved level of education. We used parents' education as a proxy for socioeconomic status (SES). The overall mean for parents' self-reported level of education was $5.30(\mathrm{SD}=1.83)$, just above high school completion. The immigrant parents on average had finished secondary school or high school $(M=5.00 ; \mathrm{SD}=1.83)$, whereas the national parents on average had some years of college education $(M=5.98$; $\mathrm{SD}=1.83$ ).

Given that the mock peers and mock parents were all randomly picked within group or within country and none was selected twice, their distributions in terms of SES, age, and gender were identical. Parental participation rates in some countries were very low for both immigrants (e.g., 19 parents in Germany) and nationals (e.g., 24 parents in France). Given these sampling limitations, our primary focus was not on country or ethnic group comparisons, but rather on the transmission of family values in national and immigrant families irrespective their country of residence.

Not all adolescents who participated in the original ICSEY study were included in the analyses for this article: we included only those whose parents also participated. This raises the question whether or not the eventual sample was selective with respect to origin (immigrant or national), gender, age, or length of residence. The proportion of adolescents with a participating parent was slightly higher for immigrant adolescents (49.4\%) than for national adolescents $(44.7 \%)$, and slightly higher for girls
$(50.8 \%)$ than boys $(44.5 \%)$. The average age of the adolescents with a participating parent was very similar to that of the other adolescents (15.29 and 15.35 years, respectively). No differences were found either in generational status or in the length of residence between immigrant adolescents with and without a participating parent. In short, in terms of demographic characteristics the analytic sample that we used was largely comparable to the original ICSEY sample.

\section{Measures}

A questionnaire assessed a wide range of variables related to acculturation and adaptation. Here we present only those that were used for the present study.

\section{Family Obligations}

This scale (10 items) describes adolescents' acceptance of their obligations and responsibilities toward their parents. This is a sample item: "Children should obey their parents." Adolescents and parents answered identical questionnaire items. They indicated on a five-point Likertscale their agreement with the statements $(1=$ "strongly disagree", $5=$ "strongly agree"). Cronbach's alphas were .77 for the immigrant adolescents and .75 for the immigrant parents. In the national sample, the corresponding alphas were .73 and .74. We also examined whether the obligations scale measured the same psychological construct (obligations) in all cultural groups in all countries using a procedure described by Van de Vijver and Leung (1997). The scale was unidimensional and we found very strong support for its structural equivalence across cultures (for further information, see Vedder and Van de Vijver 2006).

\section{Demographic Variables}

Three other variables were used to assess the broader ecological setting in which value transmission takes place: respondents' gender, parents' level of education [an eight point rating scale running from no primary school to (post) graduate degree], and immigrant adolescents' proportion of life spent in the country of residence.

\section{Zeitgeist}

In the present study, we used two definitions of Zeitgeist. For both definitions, we added to each adolescent case a peer (mock peer) and a parent (mock parent). In the first definition (the ethnic Zeitgeist) peers were randomly chosen from within the same-ethnic group (within country). We did the same for the mock parents (independent 
of the choice of the mock peer), but we made sure that the mock parent had the same sex as the adolescent's actual parent who completed the questionnaire. Together, these mock peers and mock parents represented the ethnic Zeitgeist. For the second definition of the Zeitgeist (national Zeitgeist), we followed the same procedure, but this time the mock peers and mock parents were sampled from within the same country. We made sure that within a case the mock peers and mock parents were independent of the parents and adolescents as well as amongst themselves. This means that we tried to randomize all kinds of possible dependencies in the original data set due to such factors as adolescents being together in class, living in the same neighborhood, or adults being each other's friends.

\section{Procedure}

Data were collected in all countries by the researchers themselves or by research assistants who where either postgraduate students or teachers and who were selected and trained by the principal investigators in each country. Research assistants were mostly members of the ethnocultural group with which they worked. Data collection involved completing a structured questionnaire. The questionnaire was self-explanatory but, when group administered, standard instructions were given at the start of the session. All participants were informed that participation was voluntary, and that responses were anonymous. Most questionnaires were group administered in classrooms. In some cases, adolescents were approached individually and the questionnaire was filled out individually. In case of supervised administration the data collectors were instructed to see that the questionnaire was completed in a quiet room, sometimes at home, in schools or at clubs. When whole classes participated, data were sometimes collected by the regular class teachers who received special instructions. In Sweden and Finland, mail surveys were used for groups with a very low regional concentration. These were generally very small groups. Parents mostly received a questionnaire in a return envelope through their child and were invited to complete the questionnaire at home and then return it to the researchers. Adolescents and parents were given some information about the project, in particular the international aspect of the study. All parents and adolescents used a national language version of the questionnaire, although ethnic language versions were available.

\section{Analyses}

To assess the first hypothesis concerning relationships between actual parents and adolescents, we calculated
Pearson correlations between adolescents and their parents. We compared correlations for parent-adolescent dyads by gender composition to explore the importance of including gender in further analyses.

The two hypotheses relating to the influence of Zeitgeist were tested using hierarchical multiple regression analyses in which either adolescents' or parents' obligation scores were the dependent variables. In the first step, we entered the corresponding parents' obligation score or the offspring's' obligation score; in the second step, we entered the corresponding obligation score of the mock peer and mock parent from their own ethnic group; in the third step, we entered the corresponding obligation score of the mock peer and mock parent from their own country of residence; and on the final step, we entered parents' level of education and the adolescents' proportion of life spent in the country of residence.

A major statistical concern for this type of analysis is the potential lack of independence of observations within each country with respect to the dependent variable. The most appropriate way to deal with this problem would be to use a general linear mixed model, but given that only ten countries are included in the data file such an analysis would lack statistical power. By way of compromise we calculate intraclass correlations (ICC; between country variance divided by the sum of the between country variance and the total variance at the individual level) for adolescents' and parents' obligation scores to clarify to what extent it would be preferable to consider each country as a separate subsample. A low intraclass correlation $(<0.20)$ indicates relatively small between country variation meaning that countries tend to be comparable as regards adolescents' and parents' obligation scores. Higher intraclass correlations indicate considerable value differences between countries, making it more important to have a look at country effects.

\section{Results}

The means of the family relationship values for the immigrant and national sample are presented in Table 2 . We also included effect sizes (Cohen's $d$; the difference between two means that are being compared, divided by the pooled estimate of the standard deviation).

In the immigrant sample, differences between females and males were small $(\geq .20$ or $\leq-.20)$ and in the national group the differences are negligible. Comparing parents' and offspring's scores leads to considerable differences (immigrants: $d=-.53$; nationals: $d=-.35$ ): adolescents reported lower obligation scores than parents. Even greater differences appear when comparing immigrants with nationals: immigrants reported higher obligation scores $(d$ 's youth $=1.01$; parents $=1.28)$. 
Table 2 Means of the family obligation values in immigrant and national sample, by gender of respondents

\begin{tabular}{llll}
\hline & $N$ & $M$ & SD \\
\hline Immigrants & & & \\
$\quad$ Daughters & 702 & 3.64 & .69 \\
Sons & 550 & 3.70 & .65 \\
Mothers & 851 & 3.93 & .66 \\
Fathers & 401 & 4.10 & .54 \\
Nationals & & & \\
$\quad$ Daughters & 406 & 2.96 & .60 \\
Sons & 320 & 3.03 & .60 \\
Mothers & 525 & 3.19 & .61 \\
Fathers & 181 & 3.23 & .61 \\
\hline
\end{tabular}

The first hypothesis is that there will be significant correlations between adolescents' and their actual parents' obligations scores (Table 3). This is a necessary first step before testing the two Zeitgeist hypotheses. These latter hypotheses should clarify explanations for this basic adolescent-parent correspondence by showing that the correlations in Table 3 are not just reflecting the transmission of obligations values between parents and offspring, but are due to other factors.

Hypothesis 1 There will be significant correlations between adolescents' and parents' scores on the value of family obligations.

The general picture is that there is a significant positive correlation of around .30 for obligation scores for all immigrants and all nationals of the four adolescent-parent dyads. Obligations correlations were generally comparable between nationals and immigrants; and the results were independent of the gender composition of the dyads. Overall the role of gender is limited. This finding leads us to conclude that, for our data, it does not make sense to continue to analyze the role of gender.

We calculated the intraclass correlations (ICC) for adolescents' and parents' obligation scores in the national and immigrant samples. They varied between .10 and .17, which is sufficiently low to warrant the conclusion that the scores are largely comparable between countries, both for immigrants and nationals and for adolescents as well as for their parents.
Hypothesis 2 The correspondence in the appreciation of family relationship values between adolescent children and their parents can be explained by Zeitgeist represented as persons who are non-kin.

Analyses were conducted for the national and immigrant group separately. The results for adolescents are presented in Table $4 \mathrm{a}$ and $\mathrm{b}$. Comparing the $R^{2}$ and the standardized $\beta$ 's for model 1 confirms what we reported earlier (Table 3). The co-variation of obligation scores between parents and their children is comparable between the immigrant and national samples. Zeitgeist added extra explained variance in both analyses, and as we expected adding Zeitgeist variables did lower the explanatory power of the actual parents' score. We used the squared partial correlation as an estimate of the unique contribution of a particular independent variable within a model. In the immigrant group, the unique contribution of the parents' obligation score to explaining adolescents' obligation scores went from .11 in model 1 to .09 in model 4. In the national sample, these values were .11 and .08 , respectively. The analyses differed with respect to the role of mock peers and mock parents as well as the role of the ethnic and national Zeitgeist. In the immigrant sample, for the explanation of adolescents' obligation scores only the two ethnic Zeitgeist variables added significantly to the explanation. Again, based on the squared partial correlation for model 4 the mock peer from the own ethnic group added almost $2 \%$ and the mock parent from the own ethnic group added less than $0.5 \%$. In the national sample, it was the mock parent that both in the ethnic Zeitgeist version and in the national version significantly added to explaining variance in adolescents' obligation scores. In both cases this amounted to about $0.5 \%$. In short, both the mock peers and the mock parents play a role, albeit not a strong one, in explaining adolescents' obligation scores and generally the ethnic Zeitgeist has a stronger contribution than the national Zeitgeist.

The role of one ecological variable (parental educational level) was generally negligible, but the other ecological variable (proportion of life spent in the country of settlement), did contribute to explaining adolescents' obligation scores $(1.5 \%)$; a larger proportion of life spent in the country of settlement corresponded to lower obligation scores.

Table 3 Family obligation values; correlations between adolescents and their parents, by gender and by group (immigrants/nationals)

\begin{tabular}{lllll}
\hline & Father/son $(n)$ & Mother/son $(n)$ & Father/daughter $(n)$ & Mother/daughter $(n)$ \\
\hline Immigrants & $.20^{*}(190)$ & $.36^{* *}(360)$ & $.24 * *(212)$ & $.38^{* *}(490)$ \\
Nationals & $.31 *(89)$ & $.40^{*}(231)$ & $.32 *(90)$ & $.28^{* *}(316)$
\end{tabular}

$* p<.05 ; * * p<.001$ 
Table 4 Summary of hierarchical regression analysis for variables predicting adolescents' obligation scores

\begin{tabular}{|c|c|c|c|c|c|c|c|c|c|c|c|c|c|}
\hline \multirow[t]{2}{*}{ Variable } & \multicolumn{3}{|c|}{ Model 1} & \multicolumn{3}{|c|}{ Model 2} & \multicolumn{3}{|c|}{ Model 3} & \multicolumn{4}{|c|}{ Model 4} \\
\hline & $B$ & SE B & $\beta$ & $B$ & SE B & $\beta$ & $B$ & SE B & $\beta$ & $B$ & SE B & $\beta$ & Partial $r$ \\
\hline \multicolumn{14}{|l|}{ (a) Immigrant group; $N=1,252$} \\
\hline Parent's obligation scores & .35 & .03 & $.33 * *$ & .32 & .03 & $.30 * *$ & .32 & .03 & $.30 * *$ & .31 & .03 & $.30 * *$ & .30 \\
\hline Mock peer (ethnic) & & & & .14 & .03 & $.14 * *$ & .14 & .03 & $.14 * *$ & .13 & .03 & $.13^{* *}$ & .14 \\
\hline Mock parent (ethnic) & & & & .07 & .03 & $.06^{*}$ & .06 & .03 & $.06^{*}$ & .06 & .03 & $.06^{*}$ & .06 \\
\hline Mock peer (national) & & & & & & & .04 & .03 & .05 & .04 & .03 & .05 & .05 \\
\hline Mock parent (national) & & & & & & & .02 & .03 & .02 & .02 & .03 & .02 & .02 \\
\hline Parental education & & & & & & & & & & -.01 & .01 & -.03 & -.03 \\
\hline$\%$ life in country & & & & & & & & & & -.26 & .06 & $-.11 * *$ & -.12 \\
\hline$\Delta R^{2}$ & & & .11 & & & .03 & & & .00 & & & .01 & \\
\hline$F$ for change in $R^{2}$ & & & $154.88 * *$ & & & $17.83 * *$ & & & 1.78 & & & $8.72 * *$ & \\
\hline \multicolumn{14}{|l|}{ (b) National group; $N=726$} \\
\hline Parent's obligation scores & .32 & .04 & $.33 * *$ & .30 & .04 & $.31 * *$ & .30 & .04 & $.30 * *$ & .29 & .04 & $.30 * *$ & .29 \\
\hline Mock peer (ethnic) & & & & .03 & .03 & .04 & .02 & .03 & .03 & .02 & .03 & .03 & .03 \\
\hline Mock parent (ethnic) & & & & .08 & .04 & $.08^{*}$ & .07 & .04 & .07 & .07 & .04 & $.07 *$ & .07 \\
\hline Mock peer (national) & & & & & & & .02 & .03 & .03 & .02 & .03 & .03 & .03 \\
\hline Mock parent (national) & & & & & & & .06 & .03 & $.07 *$ & .06 & .03 & $.07 *$ & .08 \\
\hline Parental education & & & & & & & & & & -.01 & .01 & -.02 & -.03 \\
\hline$\Delta R^{2}$ & & & .11 & & & .01 & & & .01 & & & .00 & \\
\hline$F$ for change in $R^{2}$ & & & $85.58 * *$ & & & $3.77 *$ & & & 2.58 & & & .46 & \\
\hline
\end{tabular}

$* p<.05 ; * * p<.001$

The procedure was repeated using the parents' obligation score as dependent variable. For these analyses we entered the corresponding obligation score of their own child in the first step. The consecutive steps were similar to the analyses already reported. The results are presented in Table 5a and b.

When explaining parental obligation scores in the immigrant sample, we found that adding Zeitgeist variables to the prediction model lowered the percentage of explained variance linked to adolescents' obligations; it went down from 11 to $8.8 \%$. In the final model of the immigrant sample, the ethnic mock peer added $0.8 \%$ to the explanation of variance and the ethnic mock parent added $1.3 \%$. In the explanation of the variance in the national sample, only the ethnic mock peer significantly added to the explanation $(3.8 \%)$. In addition, and in both analyses, parents' level of education also contributed to the explanation of the dependent variable: in the immigrant sample $0.4 \%$, and in the national sample $1.6 \%$. In both cases, the partial correlation was negative, meaning that higher levels of parental education coincided with lower parental obligation scores.

\section{Discussion}

The overall aim of this article was to explore the relative contributions to the inculcation of the value placed on obligations to the family among adolescents and their parents from family and from society. Family included the parents of adolescents and society included non-related adults and peers from the adolescents' own ethnic group, and from those not from their group. We tested three hypotheses. (1) There will be correspondence between the obligation values of adolescents and their actual parents. (2) The correspondence in obligation values between adolescent children and their parents can be explained by Zeitgeist, represented as persons who are non-kin. (3) The strength of this correspondence depends on the definition of Zeitgeist, whether it refers to one's own ethnic group or to the broader society, including the preferred level of adolescents' family related obligations of the national sample. Testing these hypotheses should help in knowing more about significant others who may affect adolescent children's socialization in a culturally diverse society and about how sensitive their socialization and development are to cultural diversity.

The three hypotheses were largely supported by the findings. Beyond the simple correlations in obligation value scores between adolescents and their parents, the Zeitgeist also explained youths' and parents' obligation scores and contributed to explaining the strength of the relationship between adolescents' and their parents' family relationship value preferences. These findings differed for the immigrant and national samples. In the immigrant 
Table 5 Summary of hierarchical regression analysis for variables predicting parents' obligation scores

\begin{tabular}{|c|c|c|c|c|c|c|c|c|c|c|c|c|c|}
\hline \multirow[t]{2}{*}{ Variable } & \multicolumn{3}{|c|}{ Model 1} & \multicolumn{3}{|c|}{ Model 2} & \multicolumn{3}{|c|}{ Model 3} & \multicolumn{4}{|c|}{ Model 4} \\
\hline & $B$ & SE B & $\beta$ & $B$ & SE B & $\beta$ & $B$ & SE B & $\beta$ & $B$ & SE B & $\beta$ & Partial $r$ \\
\hline \multicolumn{14}{|c|}{ (a) Immigrant group; $N=1,252$} \\
\hline Adolescent's obligations & .31 & .03 & $.33 * *$ & .29 & .03 & $.30 * *$ & .28 & .03 & $.30 * *$ & .28 & .03 & $.30 * *$ & .29 \\
\hline Mock peer (ethnic) & & & & .08 & .03 & $.08 *$ & .08 & .03 & $.08^{*}$ & .08 & .03 & $.08 *$ & .09 \\
\hline Mock parent (ethnic) & & & & .11 & .03 & $.11 * *$ & .11 & .03 & $.11 * *$ & .11 & .03 & $.11 * *$ & .12 \\
\hline Mock peer (national) & & & & & & & .00 & .02 & .00 & .00 & .02 & .00 & .01 \\
\hline Mock parent (national) & & & & & & & .04 & .02 & .04 & .04 & .02 & .04 & .04 \\
\hline Parental education & & & & & & & & & & -.02 & .01 & $-.06^{*}$ & -.06 \\
\hline$\%$ life in country & & & & & & & & & & .02 & .06 & .01 & .01 \\
\hline$\Delta R^{2}$ & & & .11 & & & .02 & & & .00 & & & .00 & \\
\hline$F$ for change in $R^{2}$ & & & $154.88 * *$ & & & $14.58 * *$ & & & 1.12 & & & 2.39 & \\
\hline \multicolumn{14}{|l|}{ (b) National group; $N=726$} \\
\hline Adolescent's obligations & .33 & .04 & $.33^{* *}$ & .30 & .04 & $.29 * *$ & .30 & .04 & $.29 * *$ & .29 & .04 & $.28 * *$ & .29 \\
\hline Mock peer (ethnic) & & & & .15 & .03 & $.19 * *$ & .15 & .03 & $.18 * *$ & .16 & .03 & $.19 * *$ & .20 \\
\hline Mock parent (ethnic) & & & & .06 & .04 & .06 & .06 & .04 & .06 & .07 & .04 & .07 & .07 \\
\hline Mock peer (national) & & & & & & & .01 & .03 & .01 & .02 & .03 & .02 & .02 \\
\hline Mock parent (national) & & & & & & & .02 & .03 & .03 & .03 & .03 & .03 & .03 \\
\hline Parental education & & & & & & & & & & -.05 & .01 & $-.12 *$ & -.13 \\
\hline$\Delta R^{2}$ & & & .11 & & & .04 & & & .00 & & & .01 & \\
\hline$F$ for change in $R^{2}$ & & & $85.58 * *$ & & & $17.58 * *$ & & & .37 & & & $12.03 *$ & \\
\hline
\end{tabular}

$* p<.05 ; * * p<.001$

sample, we found evidence that both intragenerational and intergenerational Zeitgeist effects explain adolescents' as well as parents' obligation scores, but these effects were restricted to the ethnic Zeitgeist. In the national sample, we found that only intergenerational Zeitgeist effects (mock parents) contributed to explaining adolescents' obligation scores, both in the ethnic and the national version. In this same sample, parents' obligation scores could partly be explained by the ethnic mock peers, but this latter effect did not coincide with a lowering of the initial correlation of parents' and adolescents' obligation scores. In addition, we found that the larger the proportion of life immigrant adolescents had spent in the country of settlement the lower their obligation scores were. For national and immigrant parents, we found that higher levels of parental education coincided with lower parental obligation scores. This study indeed showed an impact of peers on youths' value preference and it showed that non-kin adults have an impact. Last but not least, it showed that in an immigration context, it makes sense to distinguish the influence of one's own ethnic group from the influence of the wider community, including also other ethnic groups. There was no convincing support for Harris' contention that the peer group forms the primary environmental influence on psychological functioning (Harris 1995). We found effects of the mock peer Zeitgeist, but also of the mock parent Zeitgeist. In the national sample, we even found mock parent
Zeitgeist effects only. Apart from these Zeitgeist effects, however, when evaluating the findings from the perspective of group socialization theory it is important to keep in mind that the correlation between adolescents' and parents' scores remained substantial as proposed in our first hypothesis; this finding also qualifies the role of the peer group.

The fact that we found that proportion of life spent in the country of residence and parents' level of education added to the explanation of obligation scores beyond the contribution of Zeitgeist variables indicates that it is not just the change of the normative climate outside the family that corresponds to a lessening attitude toward family obligations. Increase in the proportion of life spent in the country of residence may indicate that children actually become more autonomous in participating in the new society. They may create more distance between their own value preferences and those preferred and represented by their parents. In a similar vein, parents' level of education seems to reflect a more liberal attitude toward adolescent obligations among more highly educated parents compared to parents with lower levels of education (cf. Schönpflug 2001).

Boehnke's research on Zeitgeist only included a national group; the present study includes both national and immigrant groups. Boehnke et al. (2007) suggest that people living in a community are dealing with a common 
Zeitgeist; this view might further suggest that immigrants and nationals living in the same community share a common Zeitgeist. We explored this by distinguishing a national Zeitgeist and a group specific or ethnic Zeitgeist. We found that the distinction matters. Reassured by this finding we further wondered whether the distinction we made is really the most important one, or would other configurations be even more influential? Boehnke's approach and the approach we adopted in this article deal with socialization processes that are linked to particular persons and particular groups that are in mutual contact and influence each other because they can be defined in geographical terms. However, these geographical limitations on a person's value preferences at the present time perhaps have lost some of their importance because of the ease with which people around the world can contact each other through the availability of new communication systems. Castells (2000) refers to this phenomenon as the culture of real virtuality and the network society. If indeed networking is so very important, then Zeitgeist influences may become broader and more fluid, more based on personal interest and personal effort to get involved with the virtual world. In short, the geographical anchors of the Zeitgeist may increasingly diminish. At the same time Castells (2004) qualifies this observed development by suggesting that a person's engagement in the global network society generates opposition. In this connection, he contrasts the space of the networks with the space of places; in these latter spaces people actively seek commonality, shelter, and belongingness that are linked to places that allow for a common experience of religion, ethnicity and nationality; in a sense, people are in actual walking distance. The existence of these opposing tendencies (sometimes termed globalization and localization) has been noted in research on acculturation (Berry 2008), where contact between groups sometimes leads not only to cultural and psychological convergence, but also to reaction, and to the reaffirmation of traditional values and customs. Hence, there is still a lot to clarify in terms of transgenerational socialization processes and the influences that play a role in these processes.

\section{Implications}

One implication of the findings, particularly the finding on the role of peers and the one on the importance of the national Zeitgeist, is that immigrant parents need to be aware of the influence non-immigrant peer friends have on their children's development. In their acculturation development hypothesis, Oppedal et al. (Oppedal 2006; Oppedal et al. 2004) have argued that the developmental trajectory an immigrant adolescent follows will depend on the groups they interact with and the kinds of social support they get from the groups. In this study, we find support for the fact that influence from outside the home is quite influential, suggesting that immigrant parents have to put in extra effort in the socialization of their children, the more time that their children spend with national peers. Perhaps it is more correct to regard the major reason for the often observed intergenerational discrepancy in family values to be due to the stronger influence that Zeitgeist has on children through group socialization, rather than on slowness on the part of immigrant parents to acquire the values of the larger society as suggested by a variety of scholars (e.g., Portes 1997; Szapocznik and Kurtines 1993).

A second implication is linked to the finding that parents' obligation preferences are affected by intergenerational Zeitgeist effects (adolescents) and not only by intragenerational effects (adult peers). Parents seem particularly sensitive to their adolescent children peers' value preferences. This may reflect parental awareness of the influence peers have on their children's value orientation and, more importantly, they seem to be adaptive to this aspect of Zeitgeist. This is more so for nationals than for immigrants. Immigrant parents seem to strike a balance with their adult peer group's value orientation.

\section{Future Directions}

Earlier, we pointed out the importance of exploring the group boundaries of influential value contexts. In this respect, we mentioned an ethnic and a national Zeitgeist and we referred to the fluid boundaries of World Wide Web contacts. Future studies should identify what group-based value preferences have an impact on persons' development. In addition, however, future studies would benefit from attempting to identify and analyze the processes that underlie inter- and intragenerational measures of correspondence in value preferences. For this latter purpose, the use of longitudinal designs is paramount. Such studies allow us to ascertain developmental changes and to explore the direction and causality of relationships that support, initiate or otherwise play a role in development.

We showed that the type of Zeitgeist, either the national or the ethnic Zeitgeist, plays a role in the strength of parent-offspring associations for the value placed on obligations. Boehnke et al. (2007) suggest that the strength of the association reflects the intensity of communication within the family to assure compliance or consensus. Combining our finding of the type of Zeitgeist with the Boehnke et al. explanation suggests that for the immigrant group the ethnic Zeitgeist is more of a challenge, leading to more communication, whereas for the national group it is both their own group Zeitgeist and the more inclusive national Zeitgeist. This may reflect that national families worry more about the wider value context, particularly 
given the immigrant communities' influences, whereas the immigrants worry more about what the value orientation of their own ethnic group is than about the possible impact of the cultural diversity in society. The presence of cultural diversity does not seem to trigger them to worry about intergenerational value transmission as much as does their belonging to their own group. These findings may reflect intergroup relationships in society (i.e., a stronger in-group bias in immigrant groups, and a more open attitude in the national groups). We did not examine these, but in order to explain this difference between immigrants and nationals it would be worthwhile to do so in future studies.

In sum, this study suggests that parents' and adolescent children's notions about the importance of family related obligations are influenced by kin as well as by non-kin representing the wider society. These findings on the whole support general socialization theories contending that there are a number of significant others who influence the socialization of children. This study helps in developing a better notion of who may function as significant others. These can be unknown, unrelated peers and adults, and in culturally diverse western societies, they are likely to be also immigrant adolescents.

Open Access This article is distributed under the terms of the Creative Commons Attribution Noncommercial License which permits any noncommercial use, distribution, and reproduction in any medium, provided the original author(s) and source are credited.

\section{References}

Aboud, F., \& Doyle, A. B. (1996). Parental and peer influences on children's racial attitudes. International Journal of Intercultural Relations, 20, 371-383. doi:10.1016/0147-1767(96)00024-7.

Berry, J. W. (2006). Cross-cultural psychology: An ecocultural approach. In Q. Jing, M. R. Rosenzweig, G. d'Ydewalle, H. Zhang, H.-C. Chen, \& K. Zhang (Eds.), Progress in psychological science around the world (pp. 229-243). Hove: Psychology Press.

Berry, J. W. (2008). Globalisation and acculturation. International Journal of Intercultural Relations, 32, 328-336. doi:10.1016/ j.ijintrel.2008.04.001.

Berry, J. W., \& Georgas, J. (2008). An ecocultural perspective on cultural transmission: The family across cultures. In U. Schönpflug (Ed.), Perspectives on cultural transmission. Cambridge: Cambridge University Press.

Berry, J. W., Phinney, J. S., Sam, D. L., \& Vedder, P. (Eds.). (2006). Immigrant youth in cultural transition: Acculturation, identity and adaptation across national contexts. Mahwah: Lawrence Erlbaum Associates.

Birman, D., Trickett, E. J., \& Vinokurov, A. (2002). Acculturation and adaptation of Soviet Jewish refugee adolescents: Predictors of adjustment across life domains. American Journal of Community Psychology, 30, 585-607. doi:10.1023/A:1016323213871.

Boehnke, K. (2001). Parent-offspring value transmission in a societal context: Suggestions for a utopian research design-with empirical underpinnings. Journal of Cross-Cultural Psychology, 32, 241-255. doi:10.1177/0022022101032002010.
Boehnke, K., Hadjar, A., \& Baier, D. (2007). Parent-child value similarity: The role of Zeitgeist. Journal of Marriage and the Family, 69, 778-792. doi:10.1111/j.1741-3737.2007.00405.x.

Bornstein, M., \& Cote, L. (Eds.). (2006). Acculturation and parentchild relationships. Mahwah: Lawrence Erlbaum Associates.

Bowes, J. M., Flanagan, C., \& Taylor, A. J. (2001). Adolescents' ideas about individual and social responsibility in relation to children's household work: Some international comparison. International Journal of Behavioral Development, 25, 60-68. doi:10.1080/01650250042000032.

Carlson, J. M., \& Iovini, J. (1985). The transmission of racial attitudes from fathers to sons: A study of blacks and whites. Adolescence, $20,233-237$.

Castells, M. (2000). The information age, Vol. 1, The rise of the network society. Oxford: Blackwell.

Castells, M. (2004). The information age, Vol. 2, The power of identity. Oxford: Blackwell.

Fuligni, A., Yip, T., \& Tseng, V. (2002). Impact of family obligation on the daily activities and psychological well-being of Chinese American adolescents. Child Development, 73, 302-314. doi:10.1111/1467-8624.00407.

Fuligni, A., \& Zhang, W. (2004). Attitudes toward family obligations among adolescents in contemporary urban and rural China. Child Development, 74, 180-192. doi:10.1111/j.1467-8624.2004.00662.x.

Georgas, J., Berry, J. W., Van de Vijver, F., Kagitcibasi, C., \& Poortinga, Y. (Eds.). (2006). Family across cultures: A 30 nation psychological study. Cambridge: Cambridge University Press.

Harris, J. R. (1995). Where is the child's environment? a group socialization theory of development. Psychological Review, 102, 458-489. doi:10.1037/0033-295X.102.3.458.

Harris, J. R. (1998). The nurture assumption: Why children turn out the way they do. New York: Free Press.

Kagitcibasi, C. (2007). Family, self and human development across cultures. Theory and applications. Mahwah: Lawrence Erlbaum Publishers.

Knafo, A., \& Schwartz, S. H. (2001). Value socialization in families of Israeli-born and Soviet-born adolescents in Israel. Journal of Cross-Cultural Psychology, 32, 213-228. doi:10.1177/ 0022022101032002008.

Kwak, K., \& Berry, J. W. (2001). Generational differences in acculturation among Asian families in Canada: A comparison of Vietnamese, Korean and East-Indian groups. International Journal of Psychology, 36, 152-162. doi:10.1080/00207590042000119.

Oppedal, B. (2006). Development and acculturation. In D. L. Sam \& J. W. Berry (Eds.), The handbook of acculturation psychology (pp. 9-112). Cambridge: Cambridge University Press.

Oppedal, B., Røysamb, E., \& Sam, D. L. (2004). The effect of acculturation and social support on change in mental health among young immigrants. International Journal of Behavioral Development, 28, 481-494. doi:10.1080/01650250444000126.

Phalet, K., \& Schönpflug, U. (2001). Intergenerational transmission of collectivism and achievement values in two acculturation contexts. The case of Turkish families in Germany and Turkish and Moroccan families in The Netherlands. Journal of CrossCultural Psychology, 32, 186-201. doi:10.1177/00220221010 32002006.

Phinney, J., Kim-Jo, T., Osorio, S., \& Vilhjalmsdottir, P. (2005). Autonomy and relatedness in adolescent-parent disagreements: Ethnic and developmental factors. Journal of Adolescent Research, 20, 8-39. doi:10.1177/0743558404271237.

Portes, A. (1997). Immigration theory for a new century: Some problems and opportunities. The International Migration Review, 31, 799-825. doi:10.2307/2547415.

Schönpflug, U. (2001). Intergenerational transmission of values: The role of transmission belts. Journal of Cross-Cultural Psychology, 32, 174-185. doi:10.1177/0022022101032002005. 
Schwartz, S. (1992). Universals in the content and structure of values: Theoretical advances and empirical tests in 20 countries. Advances in Experimental Social Psychology, 25, 1-65. doi:10.1016/S0065-2601(08)60281-6.

Szapocznik, J., \& Kurtines, W. M. (1993). Family psychology and cultural diversity. Opportunities for theory, research and application. The American Psychologist, 48, 400-407. doi:10.1037/ 0003-066X.48.4.400.

Van de Vijver, F. J. R., \& Leung, K. (1997). Methods and data analysis for cross-cultural research. Newbury Park: Sage.

Vandell, D. L. (2000). Parents, peer groups and other socializing influences. Developmental Psychology, 36, 699-710. doi:10.1037/ 0012-1649.36.6.699.

Vedder, P., \& Van de Vijver, F. (2006). Methodological aspects; Studying adolescents in thirteen countries. In J. Berry, J. Phinney, D. Sam, \& P. Vedder (Eds.), Immigrant youth in cultural transition: Acculturation, identity and adaptation across national contexts (pp. 47-70). Mahwah: Lawrence Erlbaum Ass.

\section{Author Biographies}

Paul Vedder holds chairs in Clinical Child and Adolescent Studies and in Cultural Diversity and Learning, both at Leiden University, Department of Education and Child Studies. He received his $\mathrm{PhD}$ in developmental psychology from Groningen University in the Netherlands. His research focuses on acculturation in youth and he is primarily interested in inter- and intragenerational transmission processes and in the link between acculturation processes and adaptation outcomes such as social competence, language proficiency, and the quality of interethnic relationships.
John Berry is professor emeritus of psychology at Queen's University, Kingston, Ontario. He received his $\mathrm{PhD}$ from the University of Edinburgh in 1966. He is a past president of the International Association for Cross-Cultural Psychology and has been an associate editor of the Journal of Cross-Cultural Psychology. He is particularly interested in the application of cross-cultural psychology to public policy and programs in the areas of acculturation, multiculturalism, immigration, health, and education.

Colette Sabatier is professor of developmental psychology at the University Victor Segalen in Bordeaux, France. She received her PhD from Québec University in Montreal, Canada. Her research focuses on intergenerational as well as intragenerational transmission processes that take place in acculturation contexts both in the family and in schools.

David Sam is a Ghanaian who migrated to Norway in 1984 as a student. He has a PhD in psychology and was a Visiting Fulbright Scholar to California State University in Los Angeles in 1997. He is now professor of cross-cultural psychology at the University of Bergen, Norway where he divides this position between the Schools of Psychology and of Medicine. As an immigrant, one of his research interests has been the psychology of acculturation where he has published extensively and is currently involved in studies dealing with studies on the mutual embeddedness of culture and mental health. 